\title{
Preparation of Electricity Conductive Anticorrosion Coatings For Grounding Grid
}

\author{
Tao Su${ }^{1, a}$, Zhi-guang Guo ${ }^{2, b^{*}}$ and Hong-yun $\mathrm{Li}^{2, \mathrm{c}}$, Qin-jun $\mathrm{Yu}^{1, \mathrm{~d}}$, Guo-gang \\ Wang ${ }^{3, e}$ \\ ${ }^{1}$ State Grid Sichuan Liangshan Power Supply Company,Sichuan Liangshan, 615000, China \\ ${ }^{2}$ Beijing Guowang FUDA Technology Development Co., LTD, Beijing, 100000, China \\ ${ }^{3}$ Beijing China Electrical Bright Power Technology Co., LTD, Beijing, 100000, China \\ asutao_xc@sepc.com.cn, ${ }^{b}$ guozhiguang@sdee.sgcc.com.cn, ${ }^{c}$ lihongyun@sdee.sgcc.com.cn , \\ yuqinjun_xc@sepc.com.cn, ${ }^{\mathrm{e}}$ wangguogang@sgepri.sgcc.com.cn
}

Keywords: grounding grid; epoxy resin; carbon nanotubes; coupling agent

Abstract. In the present study, new conductive and anti-corrosion coating which is suitable for grounding grid with a carbon-based conductive filler was investigated. This study determined using epoxy resin as a film forming binder, multi-walled carbon nanotubes as conductive filler, KH550 as the preferred coupling agent. According to the observation of surface performance, four point probe meter, scanning diffraction microscopy(SEM), the optimum content of carbon nanotubes was $22 \%$ (weight percentage of epoxy resin), the ratio of diluents and resin was determined to be $2.3: 1$. The obtained volume resistivity and adhesion was $7.2 \Omega$ and lever one, respectively.

\section{Introduction}

Grounding grid is an indispensable device in grid systerm for ensuring the safety of human body , devices. An excellent grounding systerm was the basic guarantee and important measure for the safe operation of grid systerm[1]. The corrosion phenomena often existed on the voltage-sharing conductors which buried underground from soil, which lead to short circuit, and brought serious threaten to the operating personnel. The voltage electricity might conduct back to control booth and breakdown the testing equipments and controlling equipments, causing huge economic losses[2,3].

Conductive anti-corrosion coating is an additional corrosion protection systerm, which painted on the surface of the carbon steel, which has preservative activity and features of "metal-like". According to the previous literatures, the served life of the steel coated with the conductive anti-corrosion coating would be prolonged to 30 years.

The major type of conductive anti-corrosion coating in our country is made by adding conductive fillers, especially conclude primary kinds of metal conductive filler. Li et al[4]. prepared the grounding coating for grid systerm with epoxy resin and silver powder, the surface resistivity decreased to $6.2 \times 10-4 \Omega . \mathrm{cm}$. Dong et al[5]. fabricated the conductive anti-corrosion coating for electrolytic industry with the raw materials of epoxy resin and glass powders coated with silver powders, the surface resistivity was $0.03-0.05 \Omega . \mathrm{cm}$. These surface resistivity of these types of conductive anti-corrosion coating withs primary filler of silver powders were dramatically reduced, but the filler content was relatively high, which increased the cost of the coating, and decreased the adhesion of coating on basic metals. Zhong et al[6]. prepared silver plated copper powders with chemical plating procedure, Lin et al[7]. Modified the copper powders with silane coupling agent, which all significantly reduced the surface resistivity, but the cost of conductive filler mainly with copper was still relatively high. The electrochemical corrosion often existed among interface of metal conductive filler of copper-silver, copper-nickel, which also promotes the corrosion.

Carbon types of conductive filler has the advantages of well conductivity, stable performance, widen sources, lower price etc. But the surface resistivity of conductive coating with traditional carbon types of conductive filler is lower than the metal types[8]. How to increase the electrical conductivity 
with less carbon types of conductive filler become the new direction of the reasearch for conductive coating.

The conductive coating used for grounding grid was investigated in this study, the selection and effection of resin matrix, conductive filler, coupling agent were the research topics. The dispersion was increased under the premise of adjusting the conductive filler, high conductivity and excellent anti-corrosion performance was balanced. The new conductive coating for grounding grid was fabricated with the surface resistivity of $7.2 \Omega . \mathrm{cm}$ and adhesion of lever 1 .

\section{Experiment}

Table. 1 the introduce of the raw material on standard and content (wt.\% of resin)

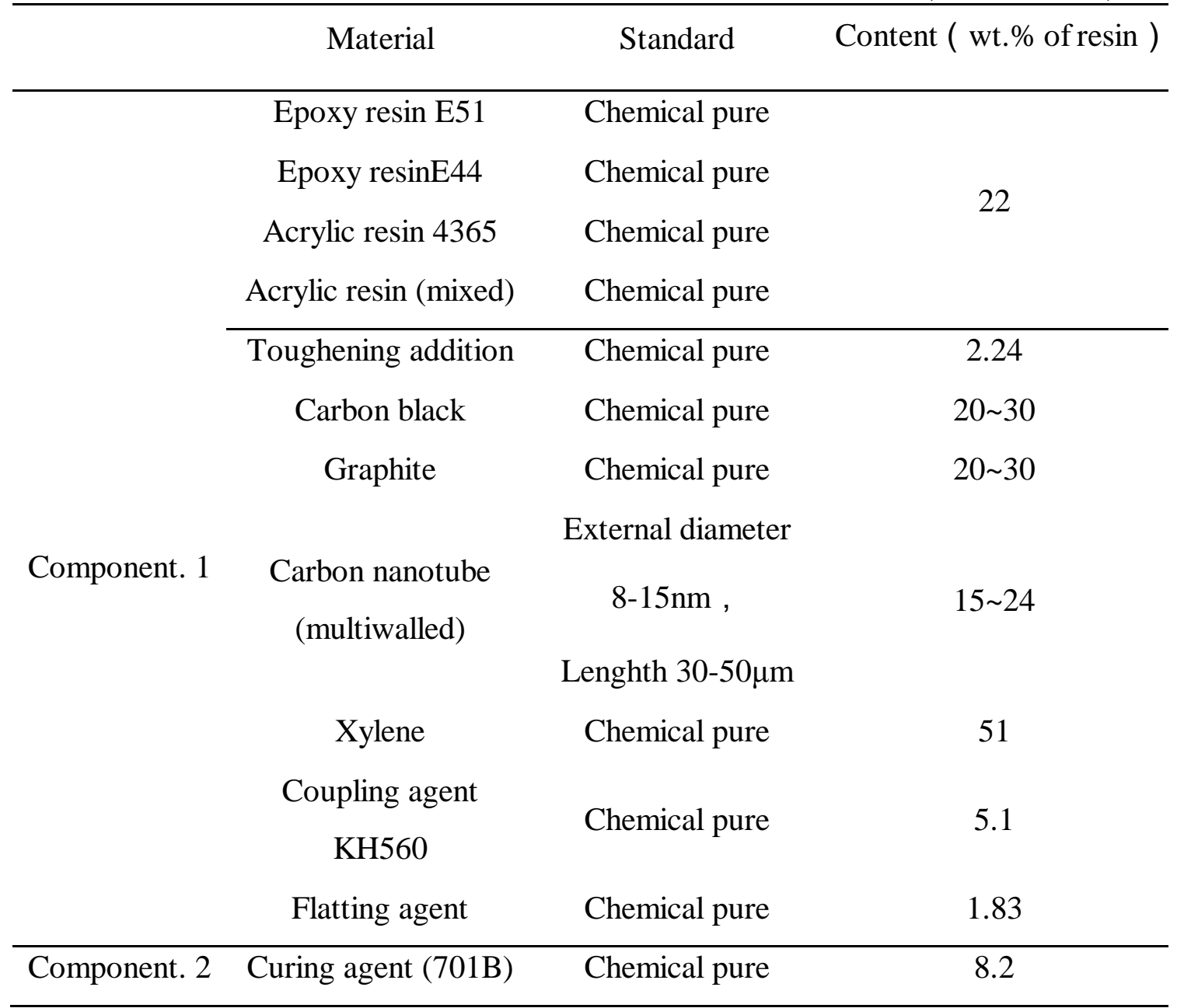

Table. 1 is the introduce of the raw material on standard and content (wt.\% of resin), it is the formula of the component. 1 and component.2.

Experimental procedures

The first step, the resin and xylene were uniformity mixed with a certain ratio, the viscosity was also adjusted, and then, the carbon nanotube, coupling agent and other agents were added into the slurry in proper order for stiring for 30min to obtain a uniform mixture. The second step, the curing agent(701B) was mix with the slurry above with the effective weight ratio of $3: 1$, the specimens were prepared by brushing the coating onto the pre-cleaned iron plate for testing.

analysis and test methods

RST-8 four-probe measuring instrument was used to test the square resistivity $R_{\square} \quad$, the thickness of the coating $(\mathrm{L})$ was measrued by the digital micrometer, surface resistivity equals the ratio of square and thickness of coating $(\mathrm{R}=\mathrm{R} \square / \mathrm{L})$.Scanning electronic microscope (SEM, Nanosem430 from FEI )was applied for observing the microstructure of the samples. Adhesion test was carried according 
GB/T 1720 - 1979.Corrosion resistance test: Painting on the precleaned steel plate, seeling the edges with paraffin, soaking for $48 \mathrm{~h}$ in sulfuric acid $(20 \mathrm{wt} . \%)$, the surface performance was tested by eye sight.

\section{Results and discussion}

Selection of resin matix

The properties of painting was decided by the structure and features of resin, which as the basic of coating. The epoxy resin and acrylic overall are the primary object in the field of conductive painting[9], which was enhanced the properties through modified and complex formulation for the broader application. Two kinds of epoxy resin and acrylic are used in this study which was evaluated by corrosion test.

Table. 2 the performances of specimens with various types of resin

\begin{tabular}{ccccc}
\hline \multirow{2}{*}{ Types of resin } & \multicolumn{2}{c}{ Adhesive force ( level ) } & \multicolumn{2}{c}{ Performance } \\
\cline { 2 - 5 } & Before corrosion & After corrosion & Before corrosion & After corrosion \\
\hline Acrylic resin-4365 & 2 & 3 & smooth , flat & Bubbling \\
Acrylic resin-mixed & 2 & 3 & smooth , flat & Bubbling, flaking \\
Epoxy resin-E44 & 1 & 2 & coarse,pitting & Pockmark,bubbling \\
Epoxy resin-E51 & 1 & 1 & coarse & unchanged \\
\hline
\end{tabular}

Table. 2 was the performances for various resin, as can be seen, the adhesive force were overall lower for acrylic resin than epoxy resin, as well as the lost of adhesive force lost after corrosion. The surface performance of the painting which use epoxy resin as the film-forming agent was coarse, for the operational environment of the painting in this study was in the soil underground, the surface performance obtained above mentioned was sytisfied for this study, corrosion resistance was the primary properties. So the epoxy resin was selected as the optimum resin in this study,

The selection and effection of conductive addition

Conductive addition was one of the key constituents in conductive coating. One complete conductive pathway should be existed in the conductive coating for obtaining electrical conductivity. So the size, morphology et al. has significant effect on the electrical conductivity for conductive painting. Graphite, carbon black, carbon nanotubes were used in this study for the electrical conductivity addition selection.

The target of electrical addition was the less addition and the maximal conductive effect. The volume of addition in this study was $15 \mathrm{wt} . \%$ (percent of epoxy resin), the surface resistivity results was listed in table 2.

Table. 3 surface resistance of painting with $15 \mathrm{wt} . \%$ electrical conduction addition

\begin{tabular}{cc}
\hline electric conduction & surface resistivity $(\Omega . c \mathrm{~m})$ \\
\hline Graphite & 120 \\
Carbon black & insulation ( or ultralimit $)$ \\
Carbon nanotubes & 40
\end{tabular}

It can be seen in the Table. 3 that the painting was of insulation(or beyound the measuring range of four-probe meter) obtained with $15 \mathrm{wt} . \%$ of carbon black addition. The surface resistivity of painting adopting with graphite was 3 times larger than carbon nanotubes for the reason of the better conductivity of carbon nanotubes. This result could be explained by the morphology of the conductive filler. Carbon black would be homogeneous dipersed in the paint matrix depends on the spherical shape, but also induced the lower contacting surface, the content of carbon black must raise to a considering quantity to obtain the better electrical conductivity. The structrure of graphite was lamellar-type, the form of contact among graphite performs "line-point" type, the broader electric circuit area was 
abtained with relative lower content of electric addition. The morphology of nanotubes was fine-rubular-type, the lap mode turened to "point-line-surface" contacting type, after dispersing in the paint, the nanotube mutually spiral wounded which generated more surface contacting area, Fig. 1 was the paint dispersed with carbon nanotubes, which clearly indicated the homogeneous dispersion state.

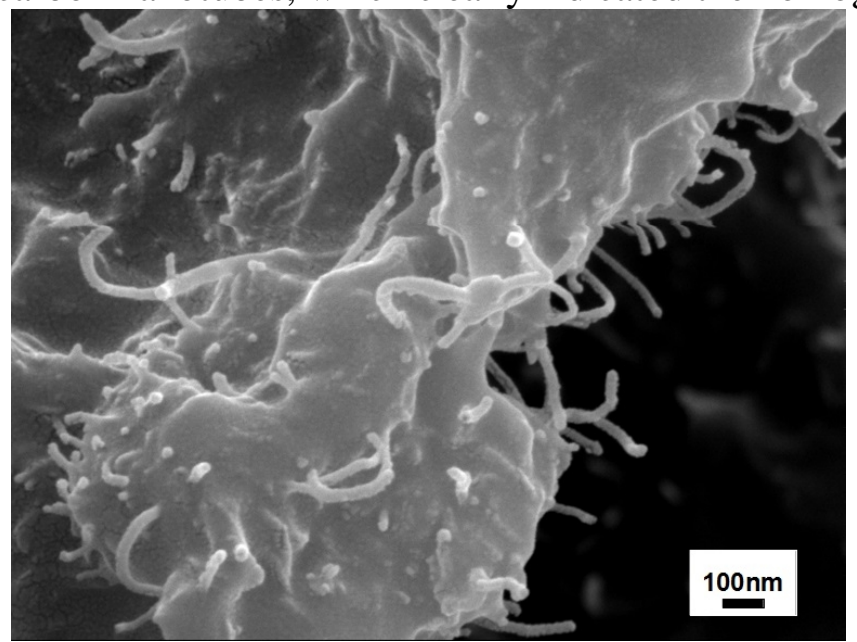

Fig. 1 The dispersed state of carbon nanotubes in epoxy resin matrix

Carbon nanotubes was selected as the conductive filler in this study according the results above, so the effect of content of conductive filler on the performance of coating was studied as follows.

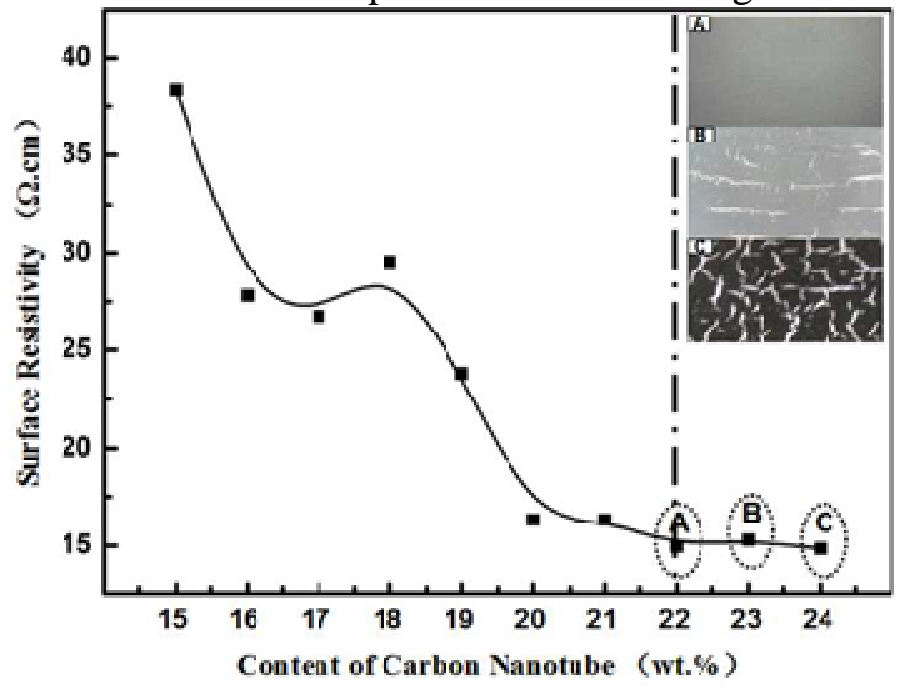

Fig. 2 Influence of carbon nanotube filler content on surface resistivity

Fig. 2 was the the influence of carbon nanotube filler content on surface resistance, it can be seen that the surface resistance of paint was decreased with the increasing of carbon nanotube filler content. The abnormal fluctuations of $18 \mathrm{wt} . \%, 21 \mathrm{wt} . \%$ might come from the difference of dispersion. Carbon nanotubes belong to inorganic filler while the epoxy resin was organics, the agglomeration enhanced the surface resistance for the bad compatibility, the performance of nano material also increased the difficulty of dispersing. After adding 22 wt.\% of carbon nanotubes, the surface resistance kept invariant, but the performance of coating surface appeared significantly changing. A large area of crack appeared on the surface of coating with the increasing of CNT filler content. It could be explained that the relative volume between epoxy resin and CNT, with the increasing of CNT, the resin was not enough to coat each CNT monomer, as well as the hollow structure of CNT would absorb the epoxy resin. The content of residual epoxy resin among the CNT was insufficient to reach the content of film-forming which lead amount of cracks. So the optimum quantity of CNT addition was $22 w t . \%$ and the surface resistance reached $15 \Omega \bullet \mathrm{cm}$.

Selection and influence of coupling agent

Carbon nanotubes belong to inorganic filler which would lead to agglomeration during mixing procedure for the bad compatibility with organic resin. The decreasing of the dispersion also reduce the 
contacting area of "point-line-surface" type of the CNT monomers, as well as reducing the service efficiency and increase the surface resistance. The bonding strength of CNT and resin decreased after filled with more CNT, which would induce the decreasing of mechanical strength and appearing the pulverization phenomenon. So it is significantly important to improve the interface of organic and inorganic[10,11].

The titanate coupling agent and silane coupling agent KH550, KH560 were selected as the coupling agent of paint in this study. The hybrid method and surface pre-treatment procedure were used to optimize the interface treatment, the effect of the coupling agent on properties of coating was also investigated.

Table. 4 Three coupling agent on coating with various procedures

\begin{tabular}{cccc}
\hline $\mathrm{a}$ & $\mathrm{b}$ & $\mathrm{c}$ & $\mathrm{d}$ \\
\hline Non-treated & Titanate & KH550 & KH560 \\
& Pre-treatment & Pre-treatment & Pre-treatment \\
& And mixed & And mixed & And mixed \\
\hline
\end{tabular}

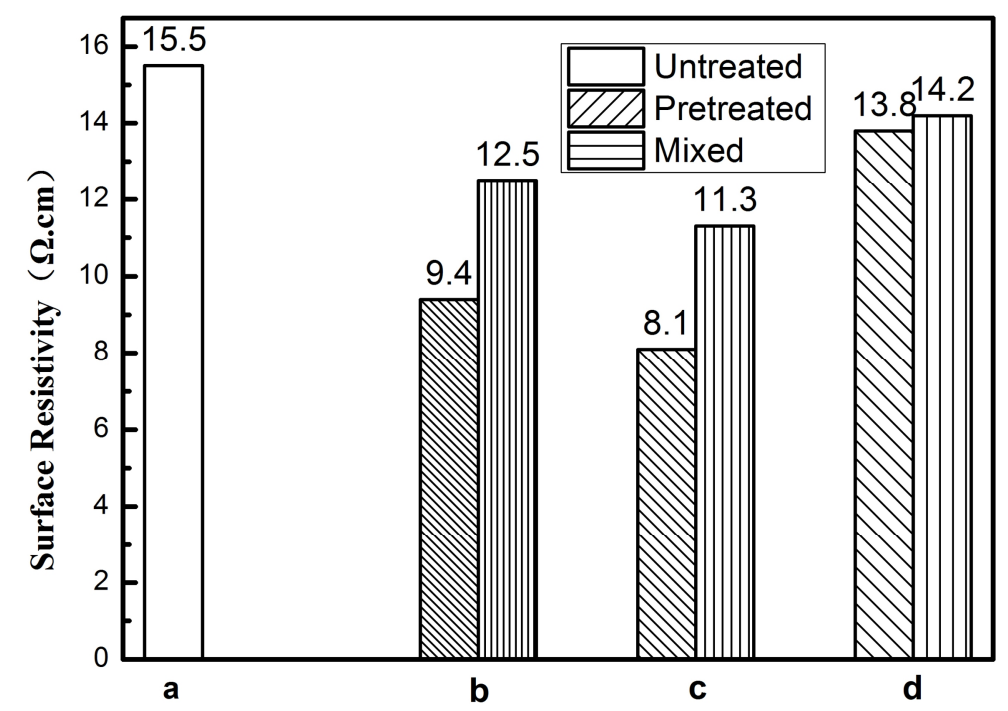

Fig. 3 Virous coupling agent and procedure on surface resistance

Table. 4 listed the three coupling agent on the coating with various procedures. Fig. 3 was the influence of various coupling agents and procedures on surface resistance properties, which can be seen that, three types of coupling agent significantly decreased the surface resistance of coating matrix, the surface resistance decreased from the three types of coupling agents, and the electrical conductivity properties of the coating which used pre-treatment was better than those use directly mixed procedure. It indicated that the pre-treatment could modified CNT, lead the organic linkers on the surface of the CNT monomers. Compared with titanate and silane, the result of silane was better than titanate which showed that the effect of surface grafting modification of silane was more significant which seemed more adapted to this painting systerm. The KH550 was the optimum selection in silane based on more amount of amino group, promoted the binding force of the CNT and epoxy resin. Fig. 3 was influence of various coupling agent and procedure on surface resistance, as shown in the Figure. 4, the dispersion of CNT modified with coupling agent KH550 was more homogeneous than the untreated specimens in the Figure. 4(a), CNT monomers could not be existed on Many parts of the paint matrix, which indicated the serious aggregation. Homodisperse CNT monomers were obtained in Fig. 4(b) with coupling agent addition, which also promoted the decreasing of surface resistivity. 

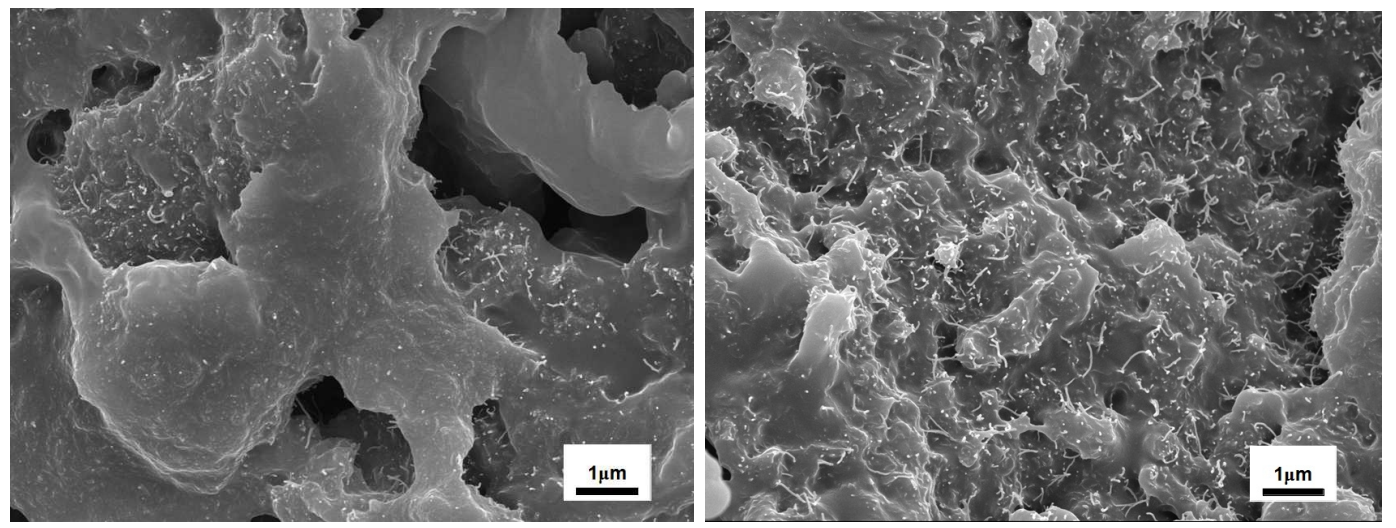

Fig. 4 Micro-morphology of paint without/with coupling agent KH550, (a) without coupling agent addition (b) with KH550 addition

Influence of diluents addition on properties of coating

The conductivity of coating was decided by the surface tension of selected resin matrix. The smaller surface tension was and the easier conductive path formed. The considering content of diluents was needed to adjusted the surface tension of the coating[12]. Under the premise of dissolving capacity of the siluent of the conductive paint, The siluent should slso have fast evaporation speed without affect the conductivity. Xylene was selected ant the siluent in this study according the precious studies. The addition amount of siluent was 1.4, 1,6, 1.8, 2, 2.2, 2.4 times of the addition of epoxy resin, respectively. The properties of the coating were also investigated in this study.

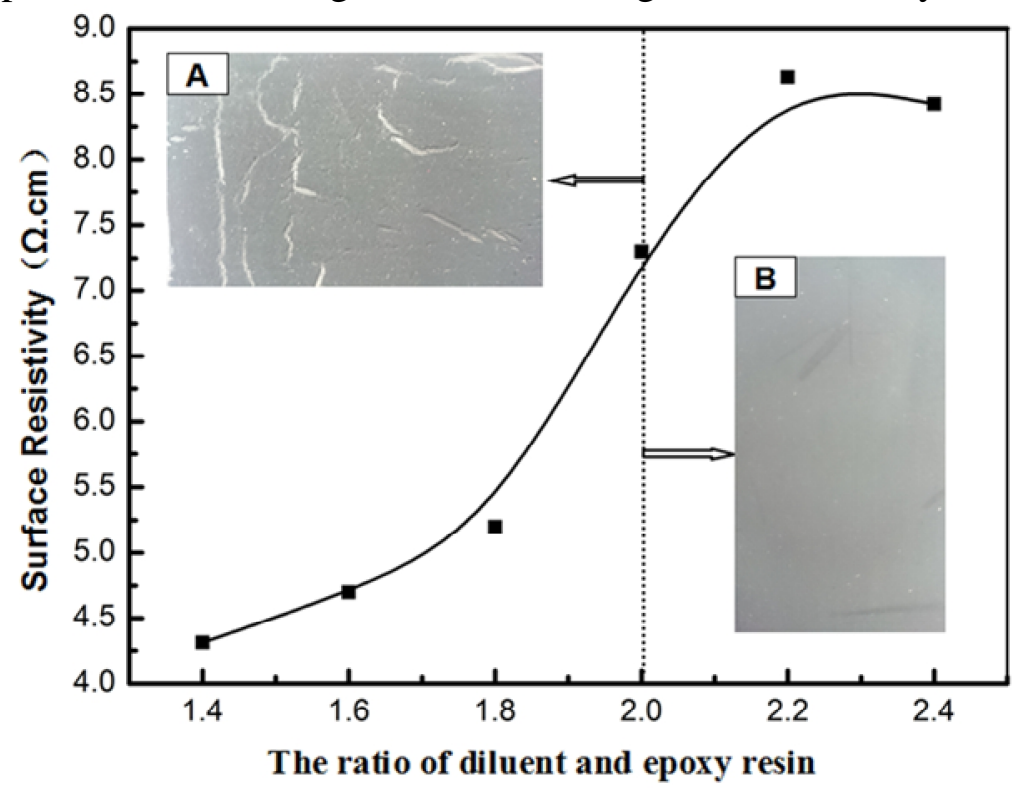

Fig. 5 Influence of siluent addition on surface resistance

Fig. 5 was the influence of siluent addion on surface resistance, which can be seen that the surface resistivity significantly increased with the decreasing of liluent addition. The surface resistivity maintained constant when and after the ratio of siluent and epoxy resin reached 2.3. According the morphology of the surface of paint, mass cracks existed before the ratio of siluent and epoxy resin arrived 2(seen in Fig. 4(a)), and also with less siluent more cracks appeared. The surface quantity turned to be smooth above the ratio 2. The existed of cracks would reduce the anticorrosion effect of the steels used for grounding grid. The diluent addition in this study was two times of the weight of epoxy resin.

\section{Conclusion}

Self-made modified epoxy resin, muti-walled carbon nanotubes was selected as the paint matrix, conductive filler, respectively. The optimum content of carbon nanotubes addition was 22 wt.\%(occupy the amount of epoxy resin) based on the conductivity and apparent condition. Silane 
coupling agent KH550 and surface pre-treated procedure was the final optimal modifier and treatment procedure according to the surface resistivity and the microstructure. The ratio of additive content of diluents and epoxy resin was 2 , which decided by the surface resistivity and apparent morphology effcted by the diluents addition. The surface resistivity of $7.2 \Omega . \mathrm{cm}$ and adhesion of level one were obtained in the final conductive coating.

\section{Acknowledgment}

This work was supported by the National Natural Science Foundation of China (Grant no. 51007083) and State Grid Technology Foundation of China (Grat no. 521700140004).

\section{References}

[1] Xiaohua Huang, Yuxue Shao et al. Corrosion and protection of substation ground network[J]. Total Corrosion Control, 2007, 21(5): 22-25.

[2] Jin Li, Hong Yang, Yishan Li. Power system grounding network research of conductive coatings [J]. Modern Paint and Finishing, 2000,9(26): 26-27.

[3] Bo Gao, Jinghua Yao, Naihong Chen et al. The development of the color of thick slurry type conducting electrostatic epoxy coating [J]. Shang Hai Coating, 2012,50 ( 02 ):5-8

[4] Jianren Shao, Yanting Le. Corrosion of grounding network communication system and protection research[J]. Electric power equipment, 2006, 7(4): 70-72.

[5] Man Dong, Xiaoyun Zhu. The preparation of conductive anticorrosive epoxy coating[J]. Electroplating and coating. 2010(09)

[6] Lianyuan Zhong, Bolin $\mathrm{Wu}$, Liyong He. Conductive coatings with flake silver plated copper powder[J], Coating industry , 2003,93(3) : 12-14

[7] Shuo Lin, Nianqiang Wu, Zhizhang Li. Composite coating conductive stability study with copper addition[J], Functional Materials[J] , 1999,30(3)

[8] Ying Li, Shifeng Wu, Yong Zhang. New black antistatic and conductive polypropylene composites filled[J]. China Plastic, 2005,33(8):39

[9] Xi Li, Jianxi Guo. The action mechanism and application of conductive coatings[J]. Tianjin Chemical Industry. 2011(03)

[10] Yongbing Dong, Baoling Zhang. The defoaming mechanism of defoaming agent and its application in leather finishing agent[J]. Chemical Engineer. 2012(02)

[11] Yuguang Zhu et al. Intrinsic type and add the performance comparison and application of conducting electrostatic anticorrosion coating[J]. Shanghai Coating, 2010, 48 ( 09 ) :19-21.

[12] Huijian Wang et al. EMI shielding conductive epoxy resin coating[D]. Taiyuan university of science and technology, 2012 